\title{
Design of a Multi-Color Plenoptic Camera for Snapshot Hyperspectral Imaging
}

\author{
Timothy W. Fahringer, $\mathrm{Jr}^{1}$ and Paul M. Danehy ${ }^{2}$ \\ NASA Langley Research Center, Hampton, VA, 23681 \\ and \\ William D. Hutchins ${ }^{3}$ \\ Virginia Tech, Blacksburg, VA, 24061
}

\begin{abstract}
The design of a custom camera lens including: a two-lens optical system, filter array, and iris has been developed enabling a greyscale plenoptic camera to acquire full field-of-view, 2D, instantaneous hyperspectral measurements. This work focuses on the use of 7 discrete color filters and their effect on the image quality. It was determined that the placement of the filters inside the aperture plane of the camera was paramount to mitigating image artifacts. In addition, design rules were developed such that the optimal optical parameters (image distance, working distance, and focal length) can be easily determined from a few charts.
\end{abstract}

\section{Introduction}

Hyperspectral or multi-spectral imaging refers to the ability to capture a 2D spectrally resolved image of scene. A review of some existing devices is given by Hagen and Kudenov. ${ }^{1}$ Typically, these systems are either instantaneous and image few wavelengths, or are time-averaged, but image a large number of wavelengths. One such instantaneous system, used for optical pyrometry, utilized a three-color $2 \mathrm{D}$ imaging system. ${ }^{2}$ Their system, and most like it, utilizes complex optical configurations to split the light into three beam paths, which pass through three different color filters, and are then imaged onto one image sensor. Adding more wavelengths to this type of system is often impractical due to the need to add more and more beam splitting elements growing the footprint of the instrument and increasing the optical complexity. A common time-averaged system involves mounting filters onto a mechanical disk, termed a filter wheel, and then imaging through each filter sequentially. The benefit of such a method is that the full image sensor can be used for each color and a large number of colors can be used (provided a large enough filter wheel); however, transient events cannot be captured and depending on the application, the failure potential of a mechanical device may be undesirable.

This work focuses on development of a 2D, instantaneous, multi-color imager based on a plenoptic camera which is a compact solution that has no moving parts. Other 2D instantaneous hyperspectral imagers exist, but are typically based on a 'Bayer' type array mounted on a traditional CCD or CMOS sensor. One such product is the PixelCam available from PIXELTEQ which can image 4 or 6 bands of the visible to NIR spectrum. The benefit of such a product is the ease of use, since it operates the same as a conventional color camera and uses off-the-shelf lenses. Our solution, however, offers greater flexibility in the selection of the optical filters in terms of wavelength and spectral width as well as the ability to easily add ND filters to balance out the signal on each channel to increase the overall dynamic range of the system. Additionally, placing the filter in the objective lens means that swapping optical filters is easy and doesn't require exposure or modification of the image sensor itself.

\section{Multi-Color Plenoptic Imaging}

The multi-color plenoptic imager is conceptually derived from the traditional plenoptic concept, which is defined by the addition of a microlens array placed one focal length in front of the image sensor such that both the spatial and angular information of the incoming light can be determined. ${ }^{3-6}$ In order for this concept to be applied as a hyperspectral

\footnotetext{
${ }^{1}$ Research Engineer, National Institute of Aerospace, AIAA Member.

${ }^{2}$ Senior Researcher, Advanced Measurement and Data Systems Branch, MS 493, AIAA Associate Fellow.

${ }^{3}$ Undergraduate Student, Department of Physics.
} 


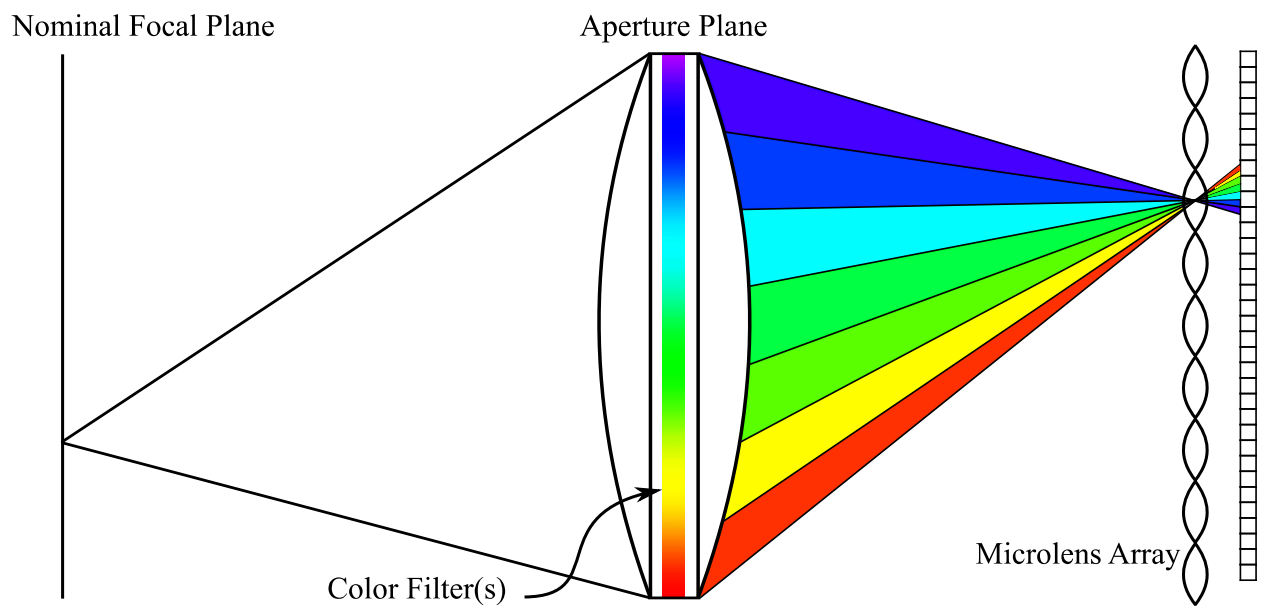

Color Filter Types

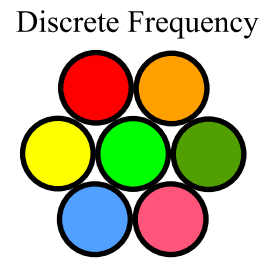

Continuous 'Rainbow' Frequency

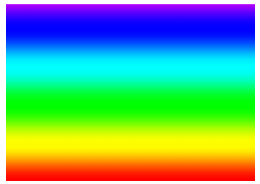

Figure 1: Hyperspectral plenoptic concept.

device a light frequency discriminator is added to the optical system. For this work, a filter, or set of filters, is added into the aperture of the camera, as shown in Figure 1Error! Reference source not found.. In a traditional plenoptic camera each sub-sample of the aperture plane is imaged onto a pixel behind each microlens as indicated by the black lines discretizing the light inside the camera. For the hyperspectral plenoptic imager, each of these sub-samples contains only a small band of the electromagnetic spectrum. The filter(s) are placed in the aperture plane, instead of the more common 'Bayer' type arrays which are added on top of the image sensor, due to the unique imaging properties of plenoptic cameras which are able to isolate sections of the aperture plane and create individual images from them, termed perspective views. It is noted that there are multiple options for the construction/layout of the filter array. The focus of this work is on using the discrete frequency color filters, as shown in Figure 1, where an array of individual circle-shaped filters are placed into a mount and then inside the aperture plane. Alternatively, a continuous, or 'rainbow' filter could be used; however this would require the added step of calibration since the frequencies that each pixel is imaging through are not well defined. In addition, the discrete filters allow for more precise selection of the frequencies/bandwidth of each filter which can be beneficial when trying to isolate specific spectral features.

\section{A. First Generation Multi-Color Plenoptic Camera}

The first generation multi-color plenoptic camera was developed by Danehy et al. ${ }^{7}$ and is shown in

Figure 2. Based on an Imperx Bobcat 6620 camera consisting of a 29 Mp CCD with 5.5 micron pixels, the plenoptic camera used in this work was created with the addition of a microlens array containing 471x362 hexagonally-arranged microlenses with a pitch of 77 microns and a focal length of 308 microns. Developed as a prototype to illustrate the concept, this camera placed three one-inch color filters $(550,632$, and $800 \mathrm{~nm})$ in front of an off-the-shelf lens to create the multi-color plenoptic imager. The focus of this work was on pyrometry and to test this instruments capabilities measurements were made on a variable-temperature blackbody source using a Mikron M360 furnace (emissivity of 0.995). After calibration for camera linearity and global intensity scaling, the temperature was determined by taking the ratio of two out of the three color channels where it was shown that these measurements had an accuracy and precision of $10 \mathrm{~K}$ for temperatures above $1100 \mathrm{~K}$. Additional data was also provided showing the capability of the instrument to resolve the $2 \mathrm{D}$ temperature distribution.

This prototype work highlighted several design considerations previously unknown. The first, and most obvious, issue was the sub-aperture image (image formed behind a microlens) was significantly smaller than anticipated. This was caused by the placement of the filter array outside the actual aperture plane as well as the choice of the main lens focal length. This had the undesirable effect of leaving large sections of the CCD unused and reduced the number of pixels/samples for each filter.

The second consideration is the presence of image artifacts caused by the placement of the filter array outside the aperture plane, as shown in

Figure 3. This figure was produced by acquiring images using the 7 filter 'discrete frequency' array shown schematically in Fig. 1 with no optical or ND filters inside the array and imaging a solid white object. Then a center perspective view was generated by selecting the center pixel behind each microlens and stitching them together to form

2

American Institute of Aeronautics and Astronautics 

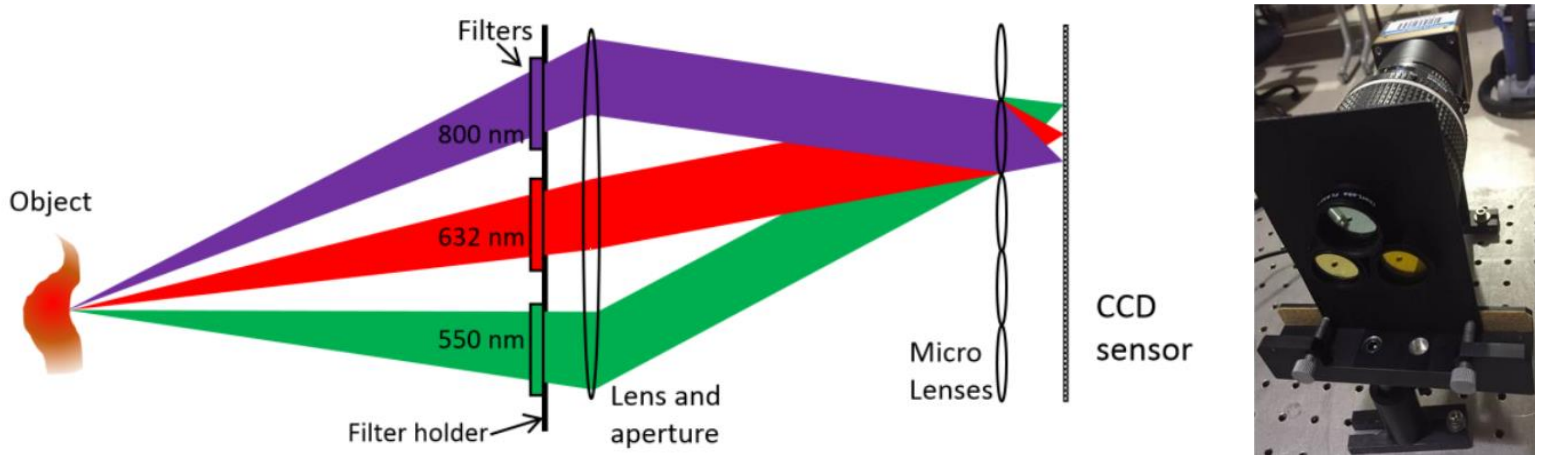

Figure 2: Schematic showing the first generation hyperspectral camera design (left) and an image of the prototype three-filter camera/filter array (right).

a single image. The strange gradients seen in the central perspective view (top) are shown to be caused by the subaperture image of the filters 'drifting' outside the microlens image. This is caused by the microlenses imaging through the filter array at an angle based on their position relative to the optical axis of the main lens. If the filter array is placed in the aperture plane the center filter will be imaged by the center of each microlens; outside this plane, however, the center filter is imaged at an angle which causes the shift in the sub aperture image as shown in the bottom row of Figure 3, where the green ' $x$ ' represents the actual center of each sub-aperture image. If the filter was located perfectly inside the aperture plane the green ' $x$ ' would lie in the center of the central filter image. Since the filter array is located outside this plane the sub-aperture image of the filter array shifts relative to the center of the microlens image. As shown in the bottom row of Figure 3, as the microlens position moves to the right, in the main image, the sub-aperture filter image is shown to shift to the left. In fact, this shift is so severe that it moves through the empty space between the filters and then eventually onto a different filter. This is why the aliasing in the top image of Figure 3 mimics the pattern of the filter array, shown as 7 white circles arranged in a hexagonal pattern, as each of the white circles is an area where the filter image has shifted such that the microlens center is imaging a different filter.

\section{Simple Lens Design}

\section{A. Basic Concept}

To reduce the artifacts presented in the previous section, a custom lens system was developed to allow the filter array to be placed in the aperture plane. The simplest form of this system consists of two lenses with the filter array placed between them, as illustrated in Figure 1. In order to hold the two lenses, allow for focusing adjustments, and an iris, a lens housing made from ThorLabs lens tubes was constructed and is detailed in Figure 4. This figure shows the placement of the focusing lens, $f_{1}$, inside a variable length lens tube allowing for precise placement in front of the microlens array inside the camera body. Then the iris and filter array are placed in the "filter plane" between the focusing and collection lens, $f_{2}$. The iris is needed in order to calibrate the microlens array relative to the image sensor, which is performed by acquiring an image while stopping down the aperture (shutting the iris) of the camera. This reveals the location of the center of each microlens image. It is noted that this diagram assumes that the light between $f_{1}$ and $f_{2}$ is collimated which is done to ensure the filter array is in the aperture plane.

\section{B. Lens Design Rules}

The use of a custom lens system allows for the precise selection and customization of main lens focal length. Therefore it is necessary to develop design rules that allow for quick and easy selection of a focal length based on desired sub-aperture image size, magnification, and working distance. The size of the sub-aperture image can be determined via geometry, based on the similarity of two triangles formed on opposite sides of the microlens by the incident light, as shown in Figure 1 where the envelope of colors formed by a common point on the microlens and the aperture and image planes respectively form the two triangles. Mathematically, the sub-aperture image can be computed by:

3

American Institute of Aeronautics and Astronautics 

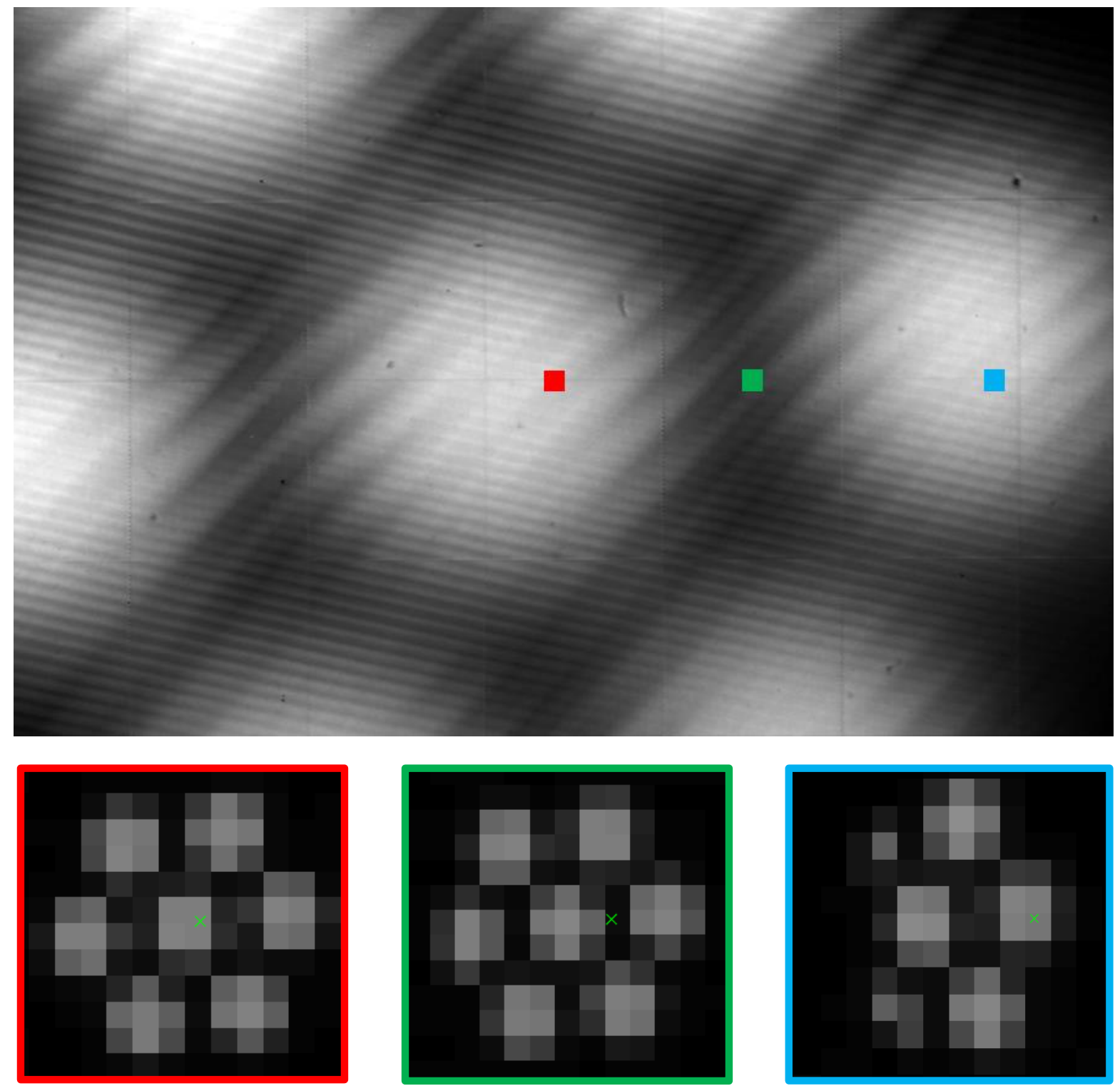

Figure 3: Perspective view image using 7 filter array (top) shows a perspective taken through the center of the aperture showing artifacts caused by the filter array being placed outside the aperture plane. Sub-images extracted from the raw plenoptic image are shown below taken at the locations indicated in the top image. The green ' $x$ ' marks the center of each microlens image. These images illustrate the 'drifting' effect of the sub-aperture images that occurs when the filter array is placed outside of the aperture plane.

$$
d_{s}=\frac{d_{a} f_{\mu}}{l_{i}}
$$

where $d_{a}$ is the aperture diameter, $f_{\mu}$ is the microlens focal length, and $l_{i}$ is the image distance. The theoretical results are shown in

Figure 5 (left) where each line represents a different aperture size. From this chart the desired image distance can be determined such that the sub-aperture image is perfectly filled. For the plenoptic camera used in this work there are 14 pixels per microlens, which corresponds to a matched sub-aperture image, and from this chart it is shown that an image distance of roughly $150 \mathrm{~mm}$ is desired for the current 7-filter design which has an aperture diameter of $38 \mathrm{~mm}$ (black 


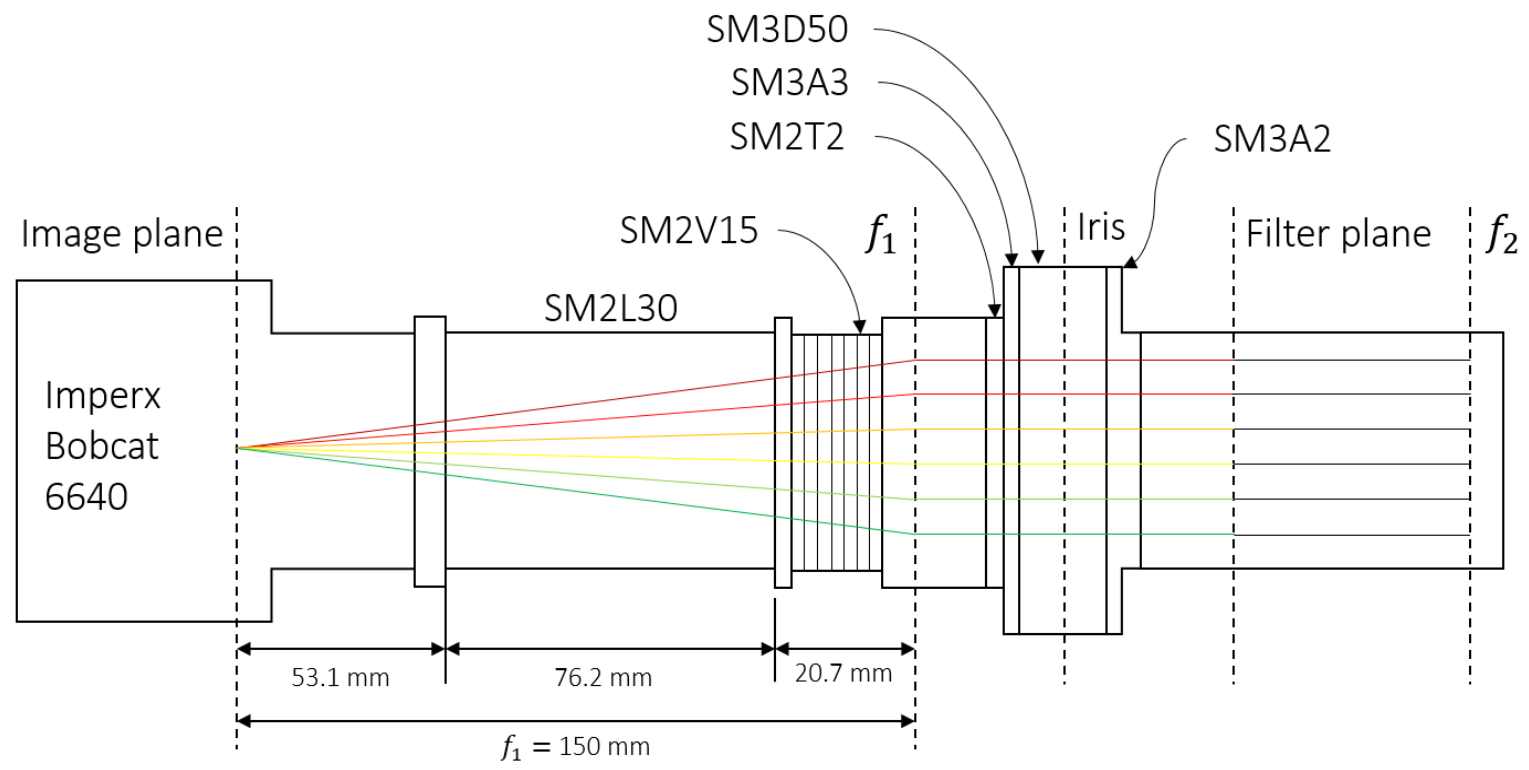

Figure 5: Schematic of camera lens assembly for $38 \mathrm{~mm}$ aperture including ThorLabs part numbers.

line). Based on the constraint of collimated light between the focusing and collection lenses the focal length of the focusing lens must be equal to the image distance and therefore $f_{1}=150 \mathrm{~mm}$.

The collection lens focal length can then be determined in one of two ways. The first is to select a desired magnification, which will yield a focal length, and working distance. The second is to select a working distance which will then yield the collection lens focal length directly and a resulting magnification. The results for selection based on working distance are shown in

Figure $\mathbf{5}$ (center) and for selection based on magnification in

Figure 5 (right). This plot shows the theoretical values, based on the thin lens equation and magnification relation. These plots are used by finding the intersection between the image distance calculated previously and the desired working distance/magnification. The result provides the effective focal length, and therefore the collection lens focal length to be used. For example if a 1:1 magnification was desired, using the current design with an image distance of $150 \mathrm{~mm}$, a roughly $80 \mathrm{~mm}$ focal length lens would be needed with a working distance of $250 \mathrm{~mm}$; however, not all combinations of working distance and magnification are possible with the current design (fixed image distance). For example, if a working distance of $750 \mathrm{~mm}$ and a magnification of 1 was desired this would not work with the current camera. Two options exist for redesigning the system to fit these needs. The first is to increase the aperture diameter such that $l_{i} / d_{a}=$
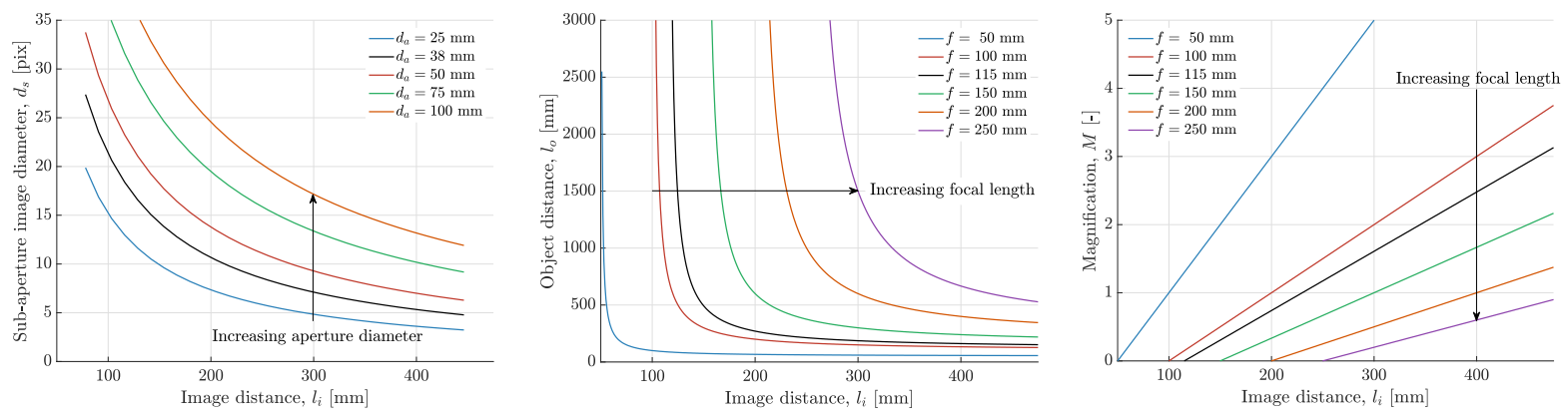

Figure 4: Design rules for hyperspectral plenoptic camera. (Left) shows sub-aperture image diameter as a function of image distance for multiple aperture diameters. (Center) shows working/object distance as a function of image distance and (right) shows magnification as a function of image distance for multiple focal lengths. Black lines indicate the configuration used in this work. 

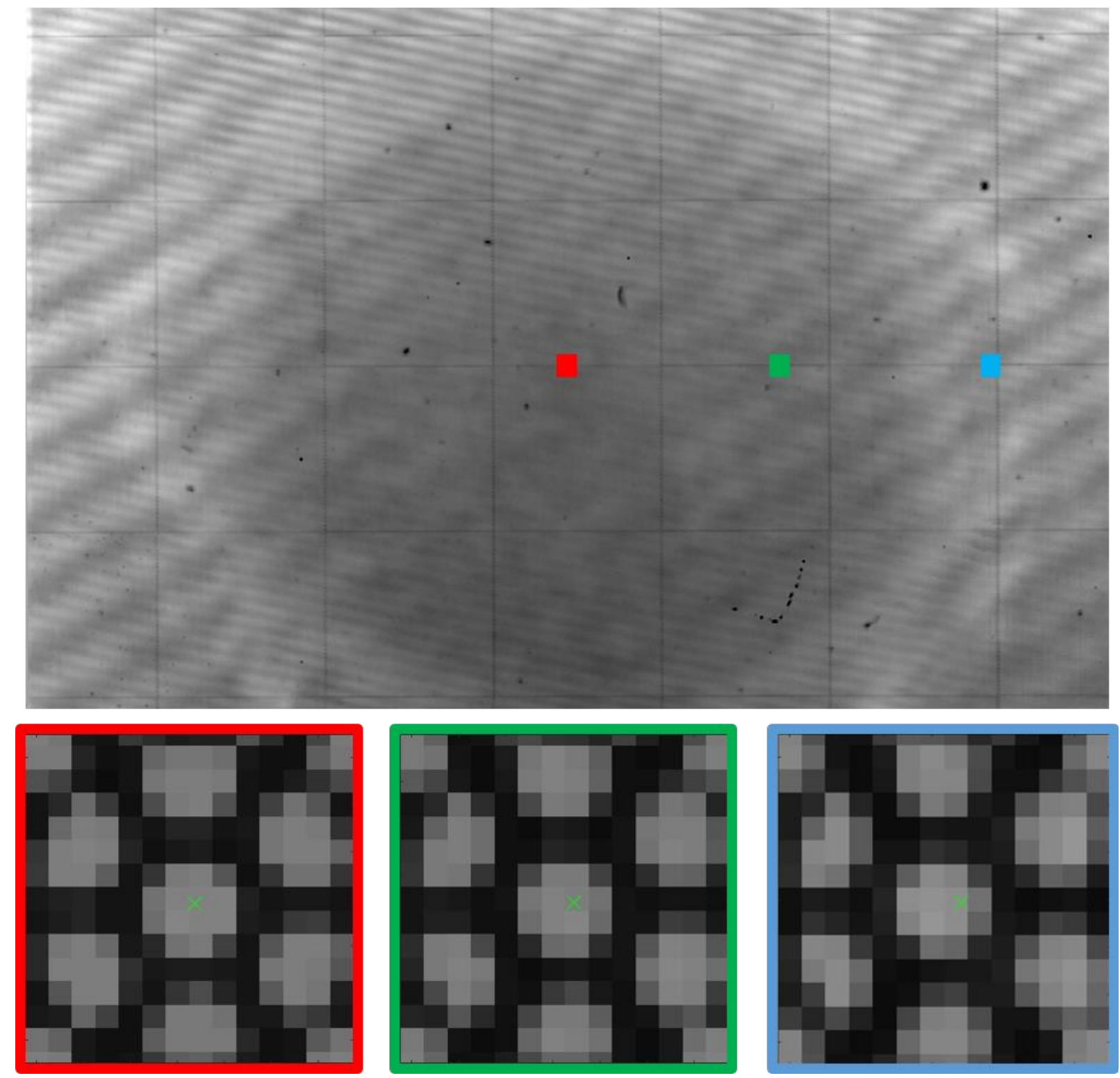

Figure 6: Perspective view image (top) shows a perspective taken through the center of the aperture using the simple custom lens housing with an effective focal length of $f_{e}=62$. 5. Sub-images extracted from the raw plenoptic image are shown below taken at the locations indicated in the top image. The green ' $x$ ' marks the center of each microlens image. These images illustrate the mitigation of the 'drifting' effect of the sub-aperture images.

$f_{\mu} / p_{\mu}$. The second is to change the $f / \#$ of the microlens array to equal $l_{i} / d_{a}$ which would involve the manufacturing of a new microlens array.

The effect of the simple lens configuration on the mitigation of the image artifacts presented in

Figure 3 are shown in Figure 6Error! Reference source not found.. This lens system drastically reduces the drifting of the sub-aperture images; however it does not fully remove this effect. In addition, it is shown that there is banding throughout the image. The further mitigation of these artifacts is the focus of the remainder of this paper.

\section{Residual Image Artifacts}

There are two types of banding present in Figure 6. The first is a larger-scale (lower spatial frequency) banding that is present at the edges of the image, most notably at the left and right sides of the image. The second is a smaller-scale 

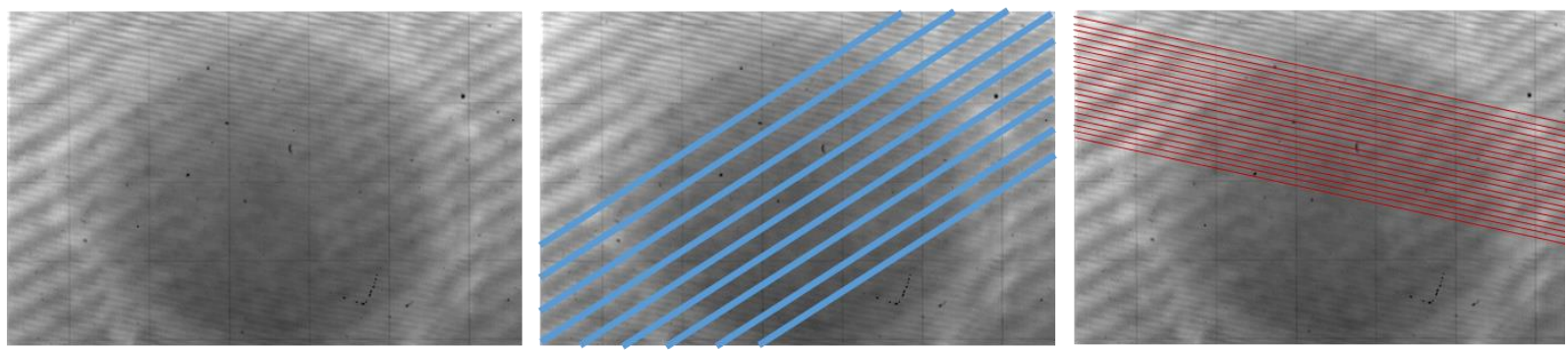

Figure 7: Illustration of residual artifacts. (left) shows original image, (center) shows larger-scale banding, and (right) shows smaller-scale banding.

(higher spatial frequency) banding that is present throughout the image, oriented in the opposite direction. Figure 7 highlights these features for clarity.

The first type of artifact (larger-scale) is created during the generation of the perspective images. Each perspective image is created by stitching together one pixel value for each microlens into a single image. Typically this is done by selecting the same pixel relative to the center of the microlens array, which is determined experimentally by stopping down the aperture of the camera and recording an image. This process creates an image where only a few pixels are illuminated behind each microlens, whose centroid is the center of the sub-aperture image. The image shown in Figure 6 is the central perspective which was created by simply stitching together the intensity at each of the center locations. An interpolation is performed to obtain a rectilinear image from a hexagonal microlens array. For typical operation where the aperture is unobstructed this works well; however, it was shown earlier that with the inclusion of the filter array the sub-aperture image moves as a function of microlens location. Therefore at the edges of the image the center of the microlens does not correspond to the center of the center filter. This is the root cause of the larger-scale banding and is why this effect is reduced near the center of the image where the drifting effect is minimal. To mitigate this effect a refinement algorithm is applied to the perspective generation technique. Using the microlens center as an initial guess, the nearest peak, above a threshold, in the sub-aperture image is selected to be used in the perspective view instead of the microlens center. For the other 6 filter positions, the same procedure is repeated but an offset is applied to the initial guess based on the design of the filter array. The results of this procedure are shown in Figure 8 where Figure 8 (left) shows the original image and Figure 8 (right) shows the refined image. It is shown that the large-scale banding has been reduced, but not quite eliminated.

The smaller-scale artifacts are caused by the imperfect alignment of the microlens array and the image sensor. Specifically, there is a slight rotation of the microlens array relative to the image sensor. The root cause of this rotation, is the fact that the alignment, both rotation as well as focus, is controlled via positioning screws that are manually adjusted. Therefore, obtaining a perfect alignment is not possible with the presently used technology, especially considering the fact that the springs unwind the screws over time. A more robust mount would solve these issues but may reduce flexibility in the design process. It is noted that the relative rotation for this case is only 0.15 degrees - not some extreme angle which could be easily corrected. In fact, the horizontal separation of the bands is the same distance that it takes for the microlenses to shift vertically by one pixel The reason this rotation causes banding is that as the images of the filters drift across the small number of pixels and occasionally the sub-aperture image aligns with a smaller group of pixels (the intersection of a $2 \times 2$ grid) and the intensity decrease for the remaining pixels, outside this region, is more severe compared to the rest of the image. Therefore, if the interpolation scheme utilizes more information (i.e. pixels) it can generate an artificially low answer by averaging artificially low pixel values when compared to regions where the sub-aperture image is spread over a $2 \times 3$ or a $3 \times 3$ region of pixels. Figure 6 was generated using bicubic interpolation to illustrate this issue. If one uses bilinear interpolation, therefore considering fewer pixels, the artifacts are mitigated, which can be seen in Figure 9 where the refined image calculated via bicubic interpolation is shown in Figure 9 (left) and calculated via bilinear interpolation in Figure 9 (right). It is also shown that the remaining larger-scale banding is also removed with in this process.

\section{Conclusions}

A study was completed to reduce image artifacts present in the first generation plenoptic hyperspectral imager. The study indicated that the root cause of the artifacts was the placement of the filter array outside the aperture plane. To mitigate these artifacts a simple lens system was developed consisting of ThorLabs lens tube elements which allowed for the placement of the filter array and an iris in between two lenses forming the lens system. This drastically reduced the sub-aperture image drifting; however residual banding artifacts remained. These residual artifacts were corrected 

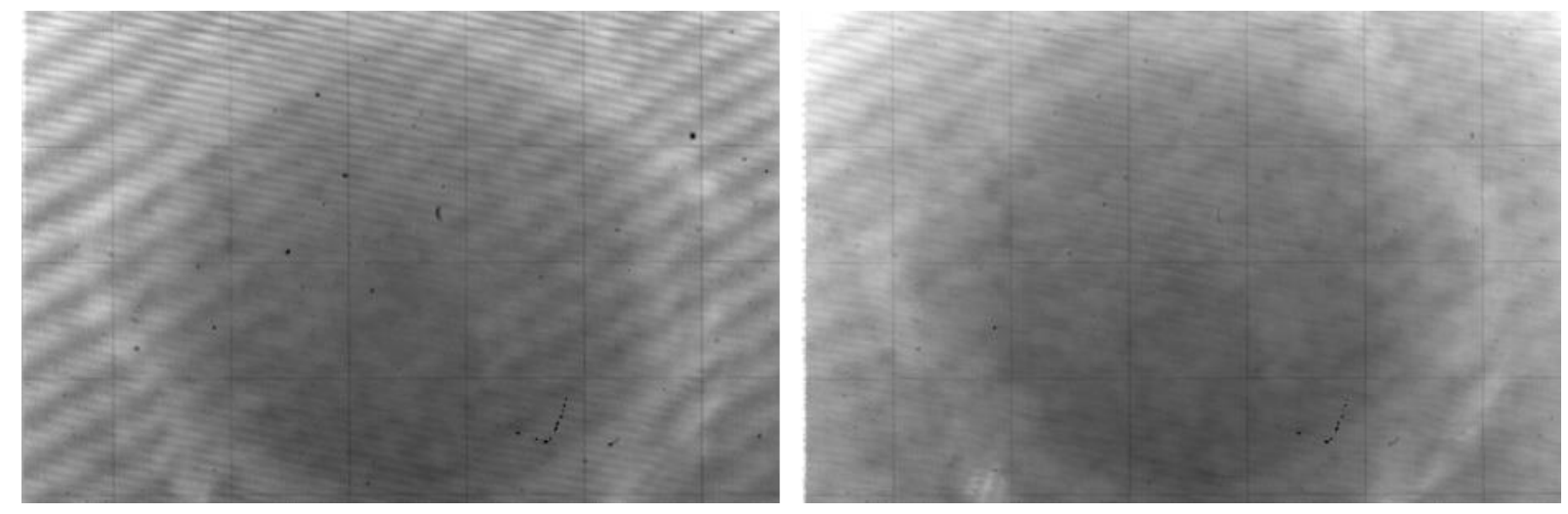

Figure 8: Illustration of the effect of the perspective generation refinement process with (left) showing the original image and (right) showing the refined image.
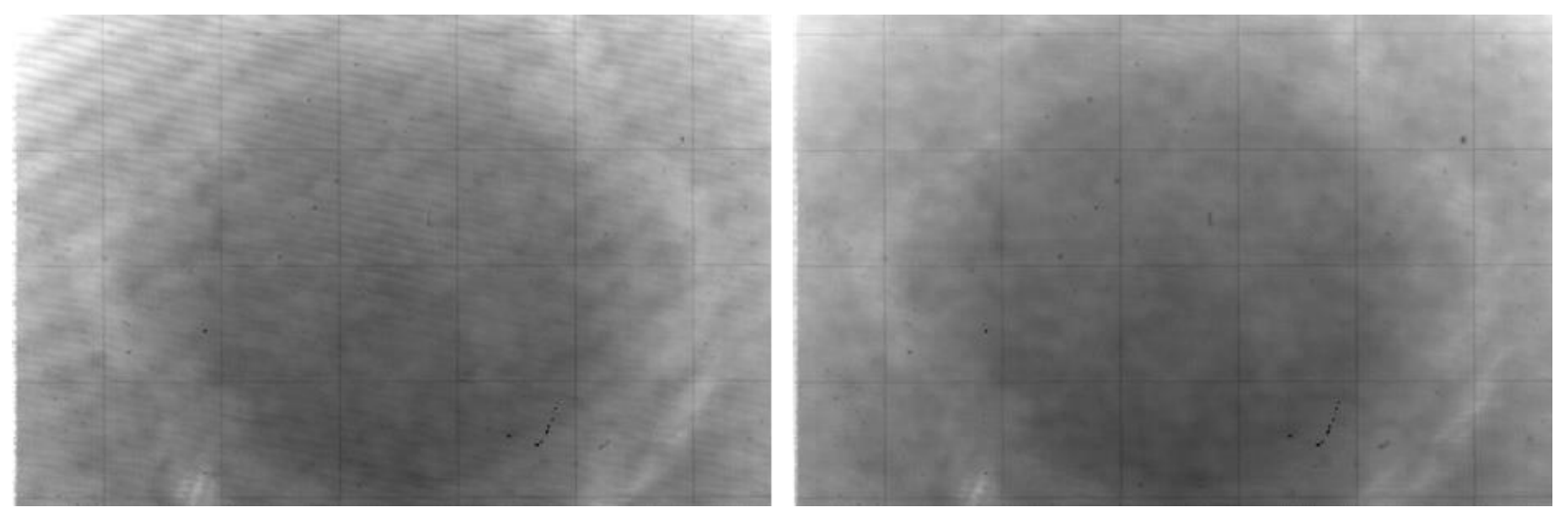

Figure 9: Illustration of the effect of cubic (left) and linear (right) interpolation on the refined image.

by refining the perspective image generation process to account for the remaining sub-aperture image drift and using linear interpolation to determine the intensity. Additionally, design rules were developed which help select the two lens elements based on a desired magnification or working distance and sub-aperture image size.

\section{Acknowledgments}

This work was supported by the NASA Langley Internal Research and Development (IRAD) program as well as the NASA Aeronautics Research Mission Directorate (ARMD) Transformational Tools and Technologies (TTT) project.

\section{References}

Hagen, N., and Kudenov, M. W., "Review of snapshot spectral imaging technologies," Optical Engineering, vol. 52, 2013, p. 90901.

Gang Lu, Yong Yan, Cornwell, S., and Riley, G., "Temperature Profiling of Pulverised Coal Flames Using Multi-Colour Pyrometric and Digital Imaging Techniques," 2005 IEEE Instrumentationand Measurement Technology Conference Proceedings, vol. 3, 2005, pp. 1658-1662.

Adelson, E. H., and Bergen, J., "The plenoptic function and the elements of early vision," Computational models of visual processing, 1991, pp. 3-20.

Adelson, E. H., and Wang, J. Y. a, "Single lens stereo with a plenoptic camera," IEEE Transactions on Pattern Analysis and Machine Intelligence, vol. 14, 1992, pp. 99-106.

5 Levoy, M., and Hanrahan, P., "Light field rendering," Proceedings of the 23rd annual conference on Computer graphics and interactive techniques - SIGGRAPH '96, 1996, pp. 31-42.

$6 \quad$ Ng, R., Levoy, M., Duval, G., Horowitz, M., and Hanrahan, P., "Light Field Photography with a Hand-held Plenoptic Camera," Informational, 2005, pp. 1-11. 
Danehy, P. M., Hutchins, W. D., Fahringer, T. W., and Thurow, B. S., “A Plenoptic Multi-Color Imaging Pyrometer," 55th AIAA Aerospace Sciences Meeting, 2017, pp. 1-7. 